\title{
Louis Maheu
}

sociologue, Département de sociologie, Université de Montréal

(1979)

\section{"Pouvoir et société au Québec : le problème de l'État et les appareils d'État”}

Un document produit en version numérique par Jean-Marie Tremblay, bénévole, professeur de sociologie au Cégep de Chicoutimi

Courriel: jean-marie tremblay@uqac.ca

Site web pédagogique : http://www.uqac.ca/jmt-sociologue/

Dans le cadre de la collection: "Les classiques des sciences sociales" Site web: http://classiques.uqac.ca/

Une collection développée en collaboration avec la Bibliothèque Paul-Émile-Boulet de l'Université du Québec à Chicoutimi

Site web: http://bibliotheque.uqac.ca/ 
Cette édition électronique a été réalisée par Jean-Marie Tremblay, bénévole, professeur de sociologie au Cégep de Chicoutimi à partir de :

\section{Louis Maheu}

"Pouvoir et société au Québec: le problème de l'État et les appareils d'État”.

Un article publié dans La transformation du pouvoir au Québec. Actes du colloque de l'Association canadienne des sociologues et anthropologues de langue française, 1979, pp. 17-26. Textes publiés sous la direction de Nadia Assimopoulos, Jacques T. Godbout, Pierre Hamel et Gilles Houle. Montréal: Les Éditions coopératives Albert Saint-Martin, 1980, 378 pp.

M Louis Maheu, sociologue, professeur au département de sociologie de l’Université de Montréal, nous a accordé le 4 janvier 2004 son autorisation de diffuser électroniquement toutes ses oeuvres.

Courriel : louis.maheu@umontreal.ca

Polices de caractères utilisée :

Pour le texte: Times New Roman, 14 points.

Pour les citations : Times New Roman, 12 points.

Pour les notes de bas de page : Times New Roman, 12 points.

Édition électronique réalisée avec le traitement de textes Microsoft Word 2004 pour Macintosh.

Mise en page sur papier format : LETTRE (US letter), 8.5'” x 11'’)

Édition numérique réalisée le 10 février 2007 à Chicoutimi, Ville de Saguenay, province de Québec, Canada. 
Louis Maheu

Sociologue, Département de sociologie, Université de Montréal
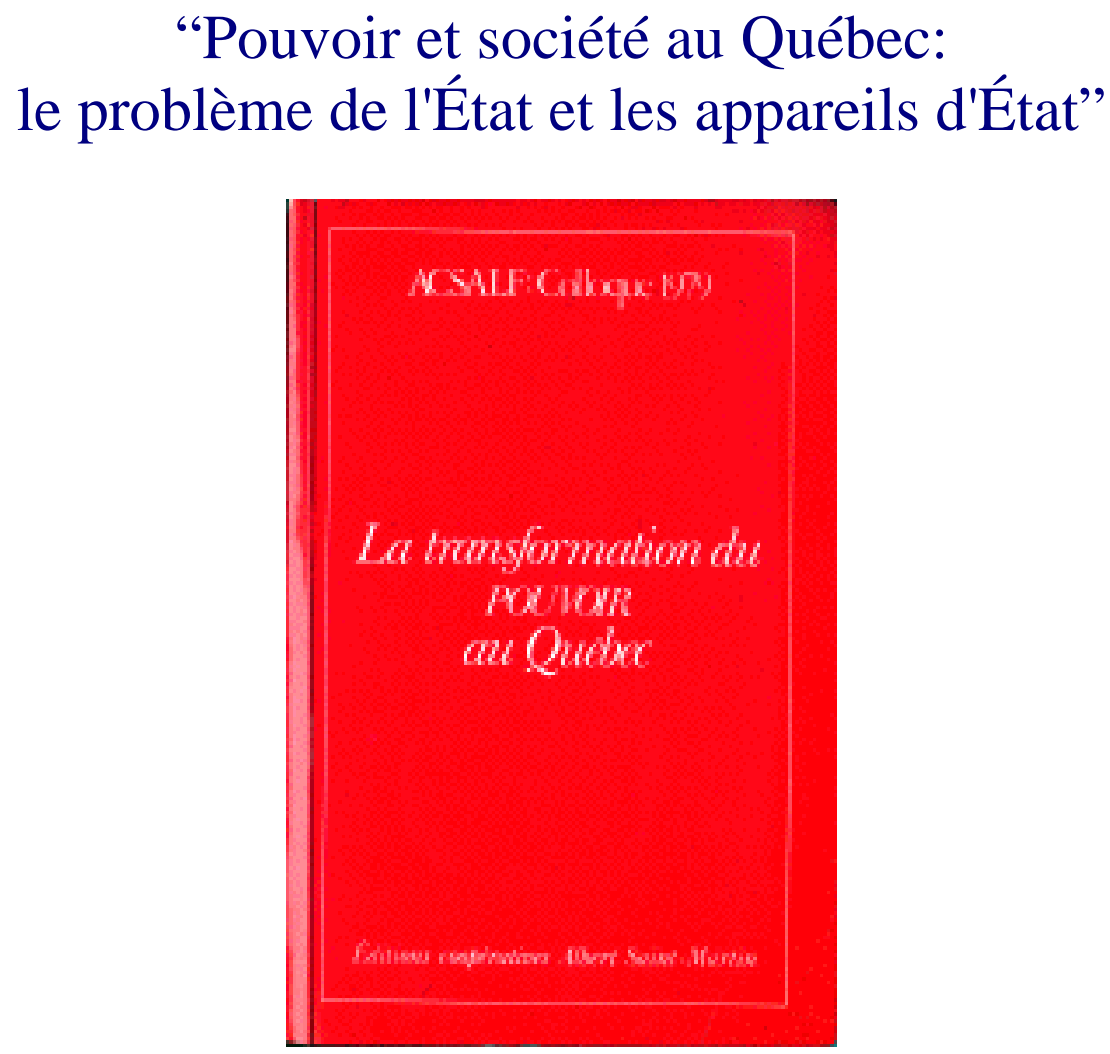

Un article publié dans La transformation du pouvoir au Québec. Actes du colloque de l'Association canadienne des sociologues et anthropologues de langue française, 1979, pp. 17-26. Textes publiés sous la direction de Nadia Assimopoulos, Jacques T. Godbout, Pierre Hamel et Gilles Houle. Montréal: Les Éditions coopératives Albert Saint-Martin, 1980, 378 pp. 


\section{Table des matières}

$\underline{\text { Introduction }}$

Technocratie et technocrates

Problèmes de dépendance

$\underline{\text { Surpolitisation des enjeux de la question nationale }}$ 
Louis Maheu

"Pouvoir et société au Québec:

le problème de l'État et les appareils d'État”.

Un article publié dans La transformation du pouvoir au Québec. Actes du colloque de l'Association canadienne des sociologues et anthropologues de langue française, 1979, pp. 17-26. Textes publiés sous la direction de Nadia Assimopoulos, Jacques T. Godbout, Pierre Hamel et Gilles Houle. Montréal: Les Éditions coopératives Albert Saint-Martin, 1980, 378 pp.

\section{Introduction}

$\underline{\text { Retour à la table des matières }}$

Je souhaite, tout d'abord, formuler quelques mises en garde qui vont, dans un sens, bien au delà de mon intention, avouée d'entrée de jeu, de ne pas tout dire à propos d'un tel thème. Je sélectionnerai donc la matière et procéderai à un découpage de l'objet qui tiendra compte d'une conception, que je souhaite défendre, du pouvoir politique. Dans une société donnée, les problèmes de l'ordre du politique sont d'abord et essentiellement fonction de la coordination, de la régulation, de l'organisation sociales globales d'une entité sociale en tant que tout capable de fonctions, de tâches plus ou moins diversifiées, complémentaires et intégrées.

On peut alors développer des problématiques du politique, ou mieux encore du politique de l'État, pour approcher ce système de rapports de forces socio-politiques - rapports de classes ou de fractions de classes sociales par exemple - tournées vers la coordination, la régulation, l'organisation sociales globales. C'est donc par rapport à ce niveau d'une problématique sociale que je parlerai tout d'abord du pouvoir. 
J'ajoute que l'on peut évidemment, en ce qui a trait aux problèmes généraux du pouvoir ou de l'organisation politique du social, distinguer d'autres niveaux, réseaux ou processus politiques articulés plus ou moins directement entre eux et avec le niveau plus global déjà identifié. Il y a d'abord le réseau des appareils politiques, soit celui des appareils de gestion de la coordination du social, qui comprend l'appareil central d'État -pouvoir exécutif et législatif, et divers appareils sectoriels d'intervention. Il y a encore les formes plus phénoménales, morphologiques du politique, soit le système des représentations politiques, des partis politiques, de l'opinion politique, des suffrages électoraux, bref, le niveau de la scène ou du marché politique au sens restreint du terme.

Le point de vue adopté va obliger à traiter de problèmes situés, le cas échéant, à l'un ou à l'autre de ces divers niveaux, tout en respectant à la fois leur grande autonomie et les mécanismes de relais et d'articulation entre eux.

Aborder le problème de cette manière n'implique pas que je soutienne que des agents principalement économiques n'aient pas de pouvoir ou encore qu'il n'y ait pas, dans une société, de pouvoirs économiques - les mêmes affirmations pouvant être faites à propos d'agents à vocation plus proprement culturelle, idéologique ou même scientifique. L'objectif est ici plutôt d'illustrer comment ces agents sociaux interviennent aussi au niveau du politique - et le cas échéant au sein d'autres réseaux des processus politiques - en tant qu'agents appartenant également à ces ensembles de rapports et s'y manifestant. En se différenciant, le niveau du politique ne devient pas étanche.

Finalement, le point de vue adopté s'oppose à une approche diffusionniste du pouvoir voulant que divers agents ou groupes d'agents sociaux aient plus ou moins de pouvoir. Le pouvoir n'est pas un continuum où divers groupes d'agents sociaux, en fonction d'une position occupée, amoncelleraient des sommes ou des masses plus ou moins imposantes ou abondantes de pouvoir. Le pouvoir fait plutôt appel à des rapports de forces socio-politiques comportant des positions plus ou moins dominantes ou plus ou moins dominées, et relatifs à des enjeux qui divisent, opposent les agents et groupes d'agents sociaux. 
Au sujet des problèmes de pouvoir au Québec, j'énonce quelques postulats qui encadrent mes propos plus qu'ils ne les structurent en parties ou sous-parties.

Au Québec, il n'y a pas à l'oeuvre, au sens strict et fort du terme, de technocratie ou de technostructure très intégrées ou à forte base localiste, ou encore de technocrates autochtones. Ce qui ne veut en rien dire qu'il n'y a pas de classe bourgeoise ou plutôt de fraction autochtone de classe bourgeoise participant à la direction économique des forces d'accumulation.

Dans la conjoncture actuelle du système des rapports de forces socio-politiques se disputant le contrôle de la coordination, de l'organisation sociales globales - donc au niveau du politique, de l'État - le poids des classes moyennes ou de fractions supérieures des classes moyennes est considérable et ne peut être négligé. Il ne s'agit aucunement, pour autant, de leur reconnaître un rôle proprement ou exclusivement hégémonique ou dominant.

Dans la conjoncture récente au Québec s'est développée une certaine forme de société civile à la croissance de laquelle les appareils d'État ont contribué et dont un trait distinctif majeur aura été l'idéologie de la participation.

En termes d'enjeux du pouvoir, la conjoncture actuelle de la société québécoise ne peut être dissociée de la question nationale, laquelle est fondamentalement de l'ordre du politique, tout en étant grandement masquée, déformée, camouflée par une certaine surpolitisation.

\section{Technocratie et technocrates}

$\underline{\text { Retour à la table des matières }}$

Au colloque de 1966 de la revue Recherches sociographiques dont il est explicitement fait mention dans la présentation de ce colloque-ci de l'ACSALF - avait été avancée l'idée d'une transformation des élites au sein de la société canadienne-française des années cin- 
quante et du début des années soixante. Une élite moderne de type technocratique aurait alors pris en main les leviers du pouvoir. Et M. Jean-Charles Falardeau n'y allait pas, en quelque sorte, avec « le dos de la cuiller », lorsqu'il soutenait qu'à cette première élite était alliée une élite économique dirigeante canadienne-française. Si la première fonctionnait à travers l'État, l'autre s'identifiait à l'entreprise et à la rue Saint-Jacques ${ }^{1}$.

Cette hypothèse, il me semble, ne peut être retenue, dans la mesure où les positions et les fonctions des technocrates sont à relier à la structuration des sociétés industrielles avancées contrôlant directement leurs destinées, appartenant aux sociétés libérales dominantes. Après les travaux de Galbraith, de Touraine et même de Bell 2- des différences considérables, bien sûr, caractérisent ces travaux et ils se distinguent notamment par ce qu'il faut y mettre un relief - on n'aurait pas de mal à soutenir que la technocratie, les agents sociaux appelés technocrates, traduisent une forme d'articulation, de rapports entre des agents économiques, politiques, intellectuels occupant les positions dominantes de la gestion du capital mais aussi de la société globale, y compris le niveau du politique, de l'État. La technocratie est donc une forme d'articulation entre fractions de classes économiquement et politiquement dominantes des sociétés libérales avancées et autocentrées.

On peut aussi trouver dans les théories marxistes contemporaines une autre expression de semblables phénomènes : les sociétés industrielles avancées, les sociétés libérales dominantes auraient vu le capitalisme monopoliste d'État succéder au capitalisme des grands mono-

1 Jean-Charles Falardeau, «Des élites traditionnelles aux élites nouvelles », dans Recherches sociographiques, vol. 7, no 1-2, 1966.

2 John Kenneth Galbraith, The New Industrial State, Boston, Houghton Mifflin Co., 1967 ; Alain Touraine, La Société post-industrielle, Paris, Denoël, 1969 ; Daniel Bell, The Coming of Post-industrial Society, New-York, Basic Books, 1973. Il existe, bien sûr, et notamment en science politique, d'autres manières d'approcher " les technocrates », que l'on tient souvent pour une élite moderne de gestionnaires de l'État, un peu comme le fait Jean-Charles Falardeau. Les propos avancés ici cherchent à se démarquer de toute approche qui traite les élites comme des agrégats d'agents sociaux isolés détachés de tout ensemble plus global de rapports entre classes et fractions de classes sociales. 
poles. Le système de rapports des forces socio-politiques (l'État) de ces sociétés pousse l'appareil central d'État, les appareils politiques, à participer à la concentration du capital et au financement de la grande production monopoliste en vue de pallier la baisse tendancielle du taux de profit, d'élargir les marchés économiques et d'assumer certaines fonctions au niveau de la dévalorisation du capital. On pourrait multiplier les traits du capitalisme monopoliste d'État ${ }^{3}$; j'en mentionne deux, assez distants de notre réalité sociale, mais qui n'en sont pas moins majeurs, principaux: la militarisation de l'économie par l'intermédiaire de l'État et le financement public d'opérations industrielles liées à la défense et à la sécurité nationales, d'une part, et la participation de l'État au développement des forces productives par son abondant financement d'un système complexe, riche, diversifié de recherche-développement, d'autre part.

Entre les analyses qui parlent de technocratie et celles qui parlent de capitalisme monopoliste d'État, il y a une parenté : elles passent par l'État. Les fractions de classes sociales dominantes de ces sociétés regroupent des agents politiques (hauts fonctionnaires de l'État, hommes politiques), économiques, intellectuels, puisque la concentration et la circulation du capital monopoliste poussent à l'articulation des monopoles et de l'État. Si la thèse de la technocratie fut appliquée au Québec, celle du capitalisme monopoliste d'État l'a été aussi : la Révolution tranquille et ses suites seraient typiques d'un État provincial appartenant à la conjoncture d'un capitalisme monopoliste d'État ${ }^{4}$.

Il est temps de dire pourquoi ces hypothèses ne me paraissent pas aptes à rendre compte des enjeux, des contraintes, du système de rapports des forces socio-politiques contrôlant l'organisation sociale globale de la société québécoise. C'est principalement que les classes économiques dirigeantes de l'industrialisation de la société québécoise sont étrangères à cette société, au sens fort (cas des bourgeoisies amé-

3 Voir à ce sujet Paul Boccara, Études sur le capitalisme monopoliste d'État, Paris, Éditions sociales, 1973, et l'ouvrage collectif Traité marxiste d'économie politique: le capitalisme monopoliste d'État, Paris, Éditions sociales, 1971, 2 tomes.

4 Diane Éthier, Jean-Marc Piotte, J. Reynolds, Les Travailleurs contre l'État bourgeois, Montréal, L'Aurore, 1975. 
ricaine et britannique) ou faible (cas de la bourgeoisie canadienneanglaise) du terme. En ce sens, le Québec n'est pas une société libérale dominante générant, contrôlant, gérant son propre développement économique. Il n'est pas dans mon propos de détailler longuement ce phénomène : beaucoup de chercheurs, ces dernières années, lui ont consacré des travaux systématiques et soignés. Je veux simplement souligner qu'on ne peut parler, à propos du Québec, d'une élite économique dirigeante autochtone forte.

\section{Problèmes de dépendance}

$\underline{\text { Retour à la table des matières }}$

Dans une telle conjoncture, comment se structure le système de rapports des forces socio-politiques contrôlant l'organisation, la coordination sociales de la société québécoise ? Il y a plusieurs voies possibles, bien sûr, mais l'analyse sociologique comparative de sociétés ainsi dépendantes amènerait à reconnaître au moins deux grands ensembles de conditions socio-politiques. Un premier ensemble met en oeuvre des bourgeoisies étrangères industrialisatrices contrôlant d'importantes unités de production dans la société locale, alliées à des classes ou fractions de classes dominantes autochtones, plutôt traditionnelles. Elles ne sont pas dès lors vraiment intéressées à une industrialisation plus accentuée ou à une modernisation de la société. Elles sont plutôt tournées vers des pouvoirs, des privilèges socio-politiques et idéologiques. Si cet ensemble de conditions socio-politiques a été déterminant pour une conjoncture antérieure de la société québécoise, ce n'est plus le cas maintenant.

Il y a donc un deuxième ensemble de conditions socio-politiques qui mène à identifier l'action de bourgeoisies économiques étrangères industrialisatrices, contrôlant toujours des unités importantes de production de la société locale, mais face auxquelles prennent position, se développent des groupes d'agents autochtones plus tournés vers l'industrialisation, le développement du marché intérieur, la modernisation de la société locale. Ces forces sociales cherchent alors à renver- 
ser les classes sociales dominantes traditionnelles, à négocier ou à imposer aux bourgeoisies étrangères leur participation à l'industrialisation. Ce dernier ensemble de conditions pourrait encore être raffiné, subdivisé, dichotomisé selon que les agents sociaux autochtones optent pour des stratégies ou contrôlent des positions leur permettant de lier la modernisation sociale recherchée à une intervention autochtone plus ou moins intensive et accentuée dans l'industrialisation et l'accumulation du capital.

Pour le moment, retenons l'essentiel : ces pratiques ou stratégies de classes dans les sociétés dépendantes sont d'abord et avant tout le fait de classes moyennes - appelées encore petite bourgeoisie, et notamment de fractions supérieures de ces classes sociales et des groupes techniques de ces classes, certains sociologues appelant ainsi les ingénieurs, les scientifiques des sciences sociales en tant qu'intellectuels modernes et, le cas échéant, les cadres supérieurs de l'armée 5.

Ces classes et fractions de classes exercent une pression telle sur le système des rapports des forces socio-politiques de ces sociétés qu'elles arrivent à influencer ou même à tenir l'appareil central d'État et ses divers appareils pour leur imposer, et les tourner vers, des politiques interventionnistes. Ces dernières visent à moderniser une société trop traditionnelle ; à contrôler la marginalité sociale de couches de population exclues ou lentes à être mobilisées par un développement économique dépendant et tardif ; à réglementer, négocier le cas échéant, les conditions d'intervention du capital étranger dans la tentative d'une quelconque préservation de l'intégrité nationale. De telles politiques interventionnistes visent encore à développer un secteur économique public intermédiaire entre les secteurs et de la bourgeoisie étrangère et de la faible bourgeoisie autochtone, pour stimuler - par l'intermédiaire de l'État - les capacités d'accumulation de capital de groupes sociaux autochtones, fortifiant, regroupant, élargissant ainsi la bourgeoisie nationale. Enfin, les politiques interventionnistes cherchent à développer la fonction et l'administration publiques, les secteurs publics d'emploi dans les appareils d'État, notamment pour les travailleurs intellectuels appartenant en général aux classes moyennes.

5 F.H. Cardoso, Sociologie du développement en Amérique latine, Paris, Anthropos, 1969. 
De là viennent les politiques qui mettent sur pied des systèmes publics d'enseignement, de santé, d'assistance sociale ; des régies d'État tournées vers l'exploitation du territoire, des ressources naturelles, vers des secteurs de production industrielle ; ou encore des programmes de gestion sociale des conflits sociaux, des conflits du travail. Bien qu'on soit là au coeur même de la conjoncture québécoise, vous aurez sans doute reconnu les lignes de force des analyses des sociétés dépendantes de Cardoso, Furtado, Martins, Ratinoff, Johnson, Touraine, Silvert ${ }^{6}$.

Je marque donc le coup en disant ceci : la Révolution tranquille et ses suites, y compris la montée sur la scène politique locale des classes moyennes et du Parti Québécois, ne nous mettent pas en présence d'une élite de technocrates ou d'un appareil central d'État gérant le développement des monopoles du capitalisme monopoliste d'État. Mais elles manifestent un système de rapports de forces socio-politiques où les pressions politiques puis économiques des bourgeoisies étrangères, de la classe ouvrière et de ses fractions s'articulent aux luttes des classes moyennes, de leurs fractions supérieures, de leurs groupes techniques, dont les visées de promotion de leurs positions et intérêts posent objectivement le problème de l'expansion, de l'élargissement d'une bourgeoisie nationale.

Et la «société civile » qu'on nous a faite dans ce processus social global et à travers les politiques interventionnistes de l'État ne porte pas que la marque - après tout, dans cette conjoncture, pas très origi-

6 F. H. Cardoso, op. cit. ; idem, Politique et développement dans les sociétés dépendantes, Paris, Anthropos, 1971 ; C. Furtado, Development and Stagnation in Latin America : A Structural Approach, New Haven, Yale University Press, 1965 ; L. Martins (éd.), Amérique latine : crise et dépendance, Paris, Anthropos, 1972 ; Alain Touraine, Les Sociétés dépendantes, Gembloux, Duculot, 1976 ; J.J. Johnson, Political Change in Latin America : the Emergence of the Middle Sectors et Continuity and Change in Latin America, Stanford, Ca., Stanford University Press, 1958 et 1964 respectivement, K.H. Silvert, The Conflict Society: Reaction and Revolution in Latin America, New Orleans, La., 1961 ; L. Ratinoff, "The New Urban Groups : The Middle Classes ", dans S.M. Lipset et A. Solari (éd.), Elites in Latin America, New-York, Oxford University Press, 1967. 
nale - de la planification, de la rationalité, de l'« expertise », mais aussi celle de la participation. En effet, les politiques de modernisation amenées par ces classes moyennes entendaient, à propos d'enjeux plus ou moins restreints comme les équipements scolaires, urbains, économiques régionaux, renouveler les interlocuteurs de l'appareil central d'État. C'est la lutte contre les fractions de classes dominantes traditionnelles, contre leurs diverses positions de pouvoir dans l'ensemble du tissu social, qui alors se poursuivait par une participationmobilisation d'agents sociaux certes plus près des classes moyennes, de leurs diverses fractions, et leur servant ainsi de groupe social d'appui.

Et même certaines couches des classes sociales défavorisées, des forces sociales contestataires, ont été objectivement associées, qu'elles l'aient voulu ou non, à la démarginalisation des exclus, des nonmobilisés, à l'intégration des agents sociaux à une société en voie de modernisation, de rattrapage. Elles ont été, plus ou moins conflictuellement, entraînées, mobilisées par un ensemble de politiques réformistes. Au point où il est difficile de voir si ces couches sociales étaient conscientes, dans leur pratique même de lutte sociale, de la mise en place de nouveaux pouvoirs de décision et d'organisation sociale que sous-tendaient les politiques interventionnistes des classes moyennes. Bref, s'il y eut diminution des écarts, rapprochement entre les groupes d'intérêt, les corps intermédiaires, la masse, le peuple et l'État, l'appareil central d'État, semblable tissage de liens sociaux plus étroits ne détruisit en rien, bien au contraire, les polarisations de classes déjà cristallisées entre positions dominantes et dominées du système de rapports de forces socio-politiques.

\section{Surpolitisation des enjeux de la question nationale}

$\underline{\text { Retour à la table des matières }}$

Un pas de plus doit être fait pour mieux saisir encore les contraintes, les enjeux du système de rapports des forces socio-politiques qui cherchent à contrôler la coordination de la société québécoise. 
Comme dans toute société dépendante, le système de rapports des forces socio-politiques de la société québécoise est ouvert sur l'extérieur. Les bourgeoisies étrangères y interviennent et les classes sociales autochtones - classes ouvrière, moyennes, bourgeoise - mènent des luttes qui ne peuvent échapper à cette contrainte. Le fonctionnement de l'appareil central d'État et de ses divers appareils est, lui aussi, ouvert sur l'extérieur.

Et, malgré des phénomènes d'occultation, même la représentation politique, la scène politique ne peuvent échapper à semblable contrainte. On pourrait alors élaborer longuement sur le morcellement qui s'ensuit, sur l'apparition des conflits sociaux ou l'éclatement de la base sociale des forces de contestation que cette situation entraîne : classe ouvrière autochtone luttant contre des forces économiques monopolistes étrangères ou contre des pouvoirs économiques locaux plus faibles, etc.

Mais dans le cas de la société québécoise, l'éclatement est encore plus accentué et exacerbé. S'il existe un système de rapports des forces socio-politiques qui - de manière prévalente, prédominante - vise le contrôle de la coordination, de l'organisation sociales de la société québécoise, les forces socio-politiques ne lui sont pas réductibles. Avec des ressources différenciées et compte tenu de leurs puissances respectives et de leurs alliances nouvelles, les forces socio-politiques interviennent aussi au niveau du système de rapports des forces sociopolitiques cherchant à contrôler la coordination de la société canadienne.

Au point où, on le sait, la forme structurelle de l'État - un appareil central canadien, des appareils centraux limités, régionaux - a été et demeure fonction de rapports de force, de luttes entre classes sociales et fractions de classes sociales regroupées régionalement et culturellement. L'état d'équilibre, plus ou moins stable, dans les relations entre les systèmes de rapports de forces socio-politiques canadien et québécois est fonction de la conjoncture des luttes entre classes sociales, fractions de classes sociales appartenant à l'un et (ou) à l'autre système. Ces contraintes marquent aussi, bien sûr, le fonctionnement des 
appareils centraux d'État et les formes plus phénoménales du politique au niveau de la scène, de la représentation politique.

Aussi, quand la conjoncture d'ensemble de la société québécoise est de l'ordre de la stimulation du marché intérieur, de la modernisation de la société, d'une industrialisation accentuée à laquelle participeraient des groupes autochtones, notamment par l'entremise de l'État et d'un secteur économique public intermédiaire - bref dans une conjoncture où le poids politique des classes moyennes, de leurs fractions supérieures, de leurs groupes techniques est considérable, ces enjeux ne concernent pas que les rapports entre classes sociales. Ou plutôt, au moyen de ces rapports entre classes et fractions de classes sociales et à travers eux, ils concernent l'état d'équilibre entre les systèmes de rapports des forces socio-politiques canadien et québécois en tant que systèmes du politique.

L'oppression nationale d'un groupe ethnique varie certes d'une classe sociale à l'autre et peut toujours être repérée à divers niveaux du social, comme les procès de production économique, les rapports à la culture, la coordination globale d'une société. La question nationale, elle, est la pratique de lutte autour d'un enjeu de l'oppression nationale qu'une classe, par ses luttes alors prévalentes, rend relativement hégémonique, prédominante, en s'appropriant conjoncturellement les intérêts de la nation.

La question nationale, dans la conjoncture actuelle, n'est pas de manière exclusive et au sens strict du terme une question de libération économique et encore moins culturelle. Elle s'adresse, au premier chef, à l'ordre du politique, de la construction de l'État, de la nation dans l'État, en tant que système de rapports de forces socio-politiques que les classes moyennes et leurs fractions supérieures cherchent à contrôler et à polariser autour de leurs intérêts, qui comprennent le maintien et l'élargissement d'une bourgeoisie canadienne-française autochtone capable de plus de dynamisme, de vigueur dans l'appropriation et l'accumulation du capital.

Bien que cet enjeu de la construction de la nation dans l'État soit prévalent dans la conjoncture actuelle, il est travesti, masqué par le fonctionnement même du système de la représentation politique, de la 
scène politique et peut-être même par celui des appareils d'État. Sont devenus thèmes électoraux les questions de l'unité canadienne, des droits constitutionnels, des gouvernements forts... À cela s'ajoutent les stratégies référendaires chapeautées, organisées par le système de la représentation politique, les partis politiques. Tout se passe comme si ces stratégies visaient à ce que, dans la nation, les agents sociaux soient sérialisés, individualisés, que les citoyens politiques soient toujours « un » et indivisibles devant des options s'adressant de manière uniforme, homogène, stéréotypée à chacun d'entre eux individuellement. Il y a là surpolitisation au niveau de la scène politique, surpolitisation qui tend à masquer que le problème est notamment de l'ordre de la domination sociale, d'un système de rapports de forces sociopolitiques, de l'État. Surpolitisation qui tend à camoufler aux classes sociales des démunis, des exclus, et notamment à la classe ouvrière, que la question nationale, dans sa conjoncture actuelle, est un enjeu de luttes de classes relatif à l'oppression nationale, principalement à celle qui est matérialisée dans les positions et les intérêts de classe des classes moyennes et de leurs fractions supérieures.

\section{Louis Maheu}

Département de sociologie

Université de Montréal

Fin du texte 